\title{
GUARANTEED PERFORMANCES DESIGN VIA MODEL SETS IDENTIFICATION
}

\author{
S. Malan* M. Milanese * D. Regruto* M. Taragna * \\ * Dip. di Automatica e Informatica, Politecnico di Torino, \\ Corso Duca degli Abruzzi 24, 10129 Torino, Italy. \\ stefano.malan@polito.it,mario.milanese@polito.it, \\ diego.regruto@polito.it, michele.taragna@polito.it
}

\begin{abstract}
In this paper an integrated robust identification and control design procedure is proposed. It is supposed that the plant to be controlled is linear, time invariant, stable, possibly infinite dimensional and that input-output noise-corrupted measurements are available. The emphasis is placed on the design of controllers guaranteeing robust stability and robust performances, and on the trade off between controller complexity and achievable robust performances. First, uncertainty models are identified, consisting of parametric models of different order and tight frequency bounds on the magnitude of the unmodeled dynamics. Second, Internal Model Controllers, guaranteeing robust closed loop stability and best approximating the "perfect control" ideal target, are designed using $\mu$-synthesis techniques. Then, the robust performances of the designed controllers are computed, allowing to determine the level of model/controller complexity needed to guarantee desired closed loop performances. Copyright (C) 2003 IFAC.
\end{abstract}

Keywords: robust control, identification for control, uncertain systems.

\section{INTRODUCTION}

The typical problem a control designer has to face in most practical situations can be briefly described as follows: a physical plant is given and a control law has to be designed, able to drive the plant to reach, if possible, given performance specifications.

The classical approach consists in building a mathematical model of the plant, on the basis of available information on it (physical laws, time invariance, linearity, etc.) and of input-output measurements, and then designing a control that meets the desired performance specifications for the identified model. However, this way it is not taken into account that any identified model is only an approximation of the actual plant. Indeed, the performances that can be actually achieved on the plant may be very poor, according to the size of the modeling error, and even the closed loop stability may be missed. These problems motivated the large interest devoted to robustness issues in the last decade. Robust control methodologies aim to design controllers guaranteeing to meet the specifications not for a single nominal model, but for all models obtained by given perturbations of the nominal model. However, the size of such perturbations has to account for the modeling error, which is not known and has to be estimated using available information and actual measurements on the plant. Moreover, the nominal model and the perturbation, indicated here as uncertainty model, have to be identified in a form suitable for the robust design, thus requiring strict interaction between identification and the goal of control design.

In this paper an integrated identification and control design procedure is proposed. It is supposed that the plant $P^{o}$ to be controlled is linear, time invariant, stable, possibly infinite dimensional and that inputoutput noise-corrupted measurements are available, together with some general information on the impulse response decay rate of the plant and on the characteristics of the noise. The emphasis is placed on the design of controllers guaranteeing robust stability and

This research was supported in part by funds of Ministero dell'Università e della Ricerca Scientifica e Tecnologica under the Project "Robustness techniques for control of uncertain systems". robust performances when used on the actual plant, and on the trade off between controller complexity and achievable robust performances.

The proposed design procedure is based on the following main steps.

First, uncertainty models are identified, consisting of models $M$ of different order and frequency bounds on the magnitude of the modeling error $\Delta=P^{o}-M$. The models are selected within given classes of parametric models $M(p)$, estimating the parameter vector $\hat{p}$ minimizing the $H_{\infty}$ norm of the modeling error. The nonparametric part of the uncertainty models accounts for the unmodeled dynamics, by evaluating tight frequency bounds $W_{\Delta}(\omega)$ on their frequency response, assuring that, under the considered assumptions, the plant $P^{o}$ is within the uncertainty models.

Second, Internal Model Controllers (IMC), guaranteeing robust closed loop stability and approximating frequency domain "perfect control" ideal target, are designed for each identified model set using $\mu$ synthesis techniques. Then, the robust performances of the designed controllers, i.e., the performances that can be guaranteed for all systems belonging to the uncertainty models, are derived. The derived controllers can be further reduced by means of standard approximation techniques until the corresponding guaranteed performances are considered acceptable.

A key point in the proposed procedure is the identification of the uncertainty model. In recent years this problem has been widely studied. For an extensive list of references, see, e.g., the surveys Milanese and Vicino (1993), Mäkilä et al. (1995) and Ninness and Goodwin (1995). However, most of the papers in the literature use a nonparametric approach, leading to identified models of high order, greater or equal to the number of data. As a consequence, if the control is designed using $H_{\infty}$ robust methods, the controller complexity is quite "high". More importantly, only rough bounds are usually derived on the magnitude of the identification error, whose degree of conservativeness is unknown or quite high. Consequently, the guaranteed performances may result very poor and conservative. 
In order to overcome in a systematic way these drawbacks, here a mixed parametric and nonparametric approach is used, aimed to derive uncertainty models with low order nominal models and tight bounds on the modeling errors, along the lines of the Special Issue on System Identification for Robust Control Design, (1992), Hakvoort and Van den Hof (1995), Zhou and Kimura (1995), Giarré et al. (1997), Venkatesh and Dahleh (2001) and Milanese and Taragna (2002). In the present paper, it is considered that measurements in the time or frequency domain are available, corrupted by pointwise $\left(\ell_{\infty}\right)$ bounded errors, possibly with known deterministic-correlation noise properties.

\section{FROM DATA TO ROBUST DESIGN}

Consider a causal, linear, time-invariant, BIBO-stable, SISO-process $P^{o}$, unknown except for some noisecorrupted measurements, either in the time domain or in the frequency domain, and for some general prior information on plant memory and measurement noise.

The model based procedure for designing a robust control consists of the following three main steps:

(1) Uncertainty model set identification.

Evaluate, from the available prior information and measured data on the plant $P^{o}$ :

- a low-order parametric model $M_{n}(\hat{p})$ with transfer function of given order $n$ depending on a parameter vector $p \in \mathbf{R}^{q}$;

- a tight frequency domain bound $W_{\Delta}(\omega)$ on the transfer function magnitude of the modelling error $\Delta=P^{o}-M_{n}(\hat{p})$ guaranteeing that:

$$
\begin{aligned}
& P^{o} \in \mathcal{M}\left(M_{n}(\hat{p}), W_{\Delta}\right)= \\
& \quad\left\{M_{n}(\hat{p})+\Delta:|\Delta(\omega)| \leq W_{\Delta}(\omega), \forall \omega\right\}
\end{aligned}
$$

In the next sections, the way the previous steps can be worked out is illustrated in some details. Moreover, the subscript $n$ in $M_{n}$ is omitted when unnecessary.

(2) Robust IMC design.

Consider an Internal Model Control (IMC) structure and compute the parameter function $Q^{*}$ taking into account the "perfect control" ideal target $(1-Q M)=0$ and the robust stability constraint $\|Q \Delta\|_{\infty}<1$. Reformulating the problem in terms of robust $H_{\infty}$ performances optimization, a solution is obtained solving the following problem:

$$
\begin{gathered}
\min _{Q \in \mathcal{R} \mathcal{H}} \sup _{\infty|\Delta| \leq W_{\Delta}}\left\|\left[1-Q\left(M_{n}(\hat{p})+\Delta\right)\right] W\right\|_{\infty} \\
\text { s.t. }\left\|Q W_{\Delta}(\omega)\right\|_{\infty}<1
\end{gathered}
$$

where $W$ is a weighting function chosen on the basis of the frequency bound where "perfect control" is desired.

(3) Guaranteed closed-loop performances computation.

Calculate the guaranteed closed-loop performances, that is to say calculate the frequency bounds for the considered performance:

$$
\begin{gathered}
\underline{H}(\omega, Q, \mathcal{M}) \leq|\tilde{H}(j \omega, Q, P)| \leq \bar{H}(\omega, Q, \mathcal{M}), \\
\forall P \in \mathcal{M}\left(M(\hat{p}), W_{\Delta}^{*}\right)
\end{gathered}
$$

where

$$
\begin{aligned}
& \bar{H}(\omega, Q, \mathcal{M})=\sup _{P \in \mathcal{M}\left(M(\hat{p}), W_{\Delta}^{*}\right)}|\tilde{H}(j \omega, Q, P)| \\
& \underline{H}(\omega, Q, \mathcal{M})=\inf _{P \in \mathcal{M}\left(M(\hat{p}), W_{\Delta}^{*}\right)}|\tilde{H}(j \omega, Q, P)|
\end{aligned}
$$

and $\tilde{H}(\cdot)$ are closed-loop transfer functions of interest, like sensitivity, complementary sensitivity, etc.

\section{UNCERTAINTY MODEL SET IDENTIFICATION}

In this section it is shown how to perform the uncertainty model set identification, using sampled data measurements of the plant. A discrete-time model is looked for to approximate the discrete-time system $P^{o}$, consisting of the plant and the input and output sampling devices.

Consider the Banach space $\mathcal{P}$ of causal, singleinput single-output, linear time-invariant, discretetime, BIBO-stable dynamical processes.

Suppose that a plant $P^{o} \in \mathcal{P}$, possibly infinitedimensional, has to be identified on the basis of noise corrupted measurements and prior information on the system and the noise.

The measurements can be in the time, frequency or mixed time and frequency domain. Such an experimental information consists of a finite number $N$ of samples and can be expressed in the form:

$$
y^{N}=F_{N} h^{P^{o}}+e^{N}
$$

where $y^{N}=\left[y_{0} \ldots y_{N-1}\right]^{T} \in \mathbf{R}^{N}$ is a known vector depending on the actual measurements, $F_{N} \in$ $\mathbf{R}^{N \times \infty}$ is a known matrix indicating how the measurements depend on $P^{o}, h^{P^{o}}=\left[h_{0}^{P^{o}} h_{1}^{P^{o}} \ldots\right]^{T} \in$ $\mathbf{R}^{\infty}$ is an unknown vector containing the impulse response $\left\{h^{P^{o}}\right\}=\left\{h_{0}^{P^{o}}, h_{1}^{P^{o}}, \cdots\right\}$ of $P^{o}$, and $e^{N}=$ $\left[e_{0} \ldots e_{N-1}\right]^{T} \in \mathbf{R}^{N}$ is an unknown vector representing the measurement noise. Explicit expressions of matrix $F_{N}$ for time and/or frequency domain experiments can be found in Milanese and Taragna (2002). Let $P^{o}(z)=\sum_{k=0}^{\infty} h_{k}^{P^{o}} z^{-k}$ be the $z$-transform of $h^{P^{o}}$, while $\left\|P^{o}(z)\right\|_{\infty}=\sup _{0 \leq \omega \leq 2 \pi}\left|P^{o}\left(e^{j \omega}\right)\right|$ denotes the $H_{\infty}$ norm of $P^{o}$.

The prior information on plant $P^{o}$ is a bound on its impulse response decay rate:

$$
P^{o} \in K=\left\{P \in \mathcal{P}:\left|h_{\ell}^{P}\right| \leq L \rho^{\ell}, \forall \ell \in \mathbf{Z}_{0}^{\infty}\right\}
$$

where $L>0$ and $0<\rho<1$ are known constants, and $\mathbf{Z}_{m}^{n}$ denotes the set of integers $\ell$ such that $m \leq \ell \leq n$.

The prior information on noise $e^{N}$ is given as:

$$
e^{N} \in \mathcal{B}_{e}=\left\{\tilde{e}^{N} \in \mathbf{R}^{N}:\left\|W_{e}^{-1} A \tilde{e}^{N}\right\|_{\infty} \leq 1\right\}
$$

where $A \in \mathbf{R}^{l \times N}$ is a known matrix with $l \geq N$, $W_{e}=\operatorname{diag}\left(w_{e, 1}, \ldots, w_{e, l}\right) \in \mathbf{R}^{l \times l}$ is a known weighting matrix with $w_{e, k}>0 \forall k$, and $\left\|W_{e}^{-1} A \tilde{e}^{N}\right\|_{\infty}=$ 
$\max _{1 \leq k \leq l} w_{e, k}^{-1}\left|\left(A \tilde{e}^{N}\right)_{k}\right|$. This assumption can accommodate not only for information on maximal noise magnitude, i.e., $\left\|W_{e}^{-1} \tilde{e}^{N}\right\|_{\infty}=\max _{1 \leq k \leq N} w_{e, k}^{-1}\left|\tilde{e}_{k}\right| \leq 1$, as typically done in most of the literature, but can also account for possible information on deterministic crosscovariance or autocorrelation properties of the noise.

In the overall identification procedure, a key role is played by the Feasible Systems Set, often called "unfalsified systems set", i.e., the set of all processes consistent with prior information and measured data.

\section{Definition 1. Feasible Systems Set}

$$
F S S=\left\{P \in K:\left\|W_{e}^{-1} A \cdot\left(y^{N}-F_{N} h^{P}\right)\right\|_{\infty} \leq 1\right\}
$$

If prior assumptions on plant $P^{o} \in K$ and on noise $e^{N} \in \mathcal{B}_{e}$ are "true", this set includes $P^{o}$, an important property in view of subsequent use for robust control design. As required in any identification theory, the problem of checking the validity of priors arises. Indeed, the only thing that can be actually done is to check if measured data falsify the priors. This is equivalent to check if $F S S$ is empty, being the $F S S$ the set of unfalsified systems. Necessary and sufficient conditions for such a check are given in Milanese and Taragna (2000). The FSS can be considered an uncertainty model set for $P^{o}$ and, in the line with the robustness paradigm, control should be designed to be robust versus such an uncertainty model set. However, the $F S S$ is not represented in a suitable form to be used by robust control design techniques, and model sets with such a property have to be looked for. Moreover, to be consistent with robust control design philosophy, uncertainty model sets including the set of unfalsified systems have to be looked for. This is formalized by the following definition.

Definition 2. Uncertainty model set

A set of models $\mathcal{M} \subseteq \mathcal{P}$ is a model set for $P^{o}$ if:

$$
\mathcal{M} \supseteq F S S
$$

In this paper, additive frequency shaped model sets are considered, of the form:

$$
\begin{aligned}
& \mathcal{M}\left(M(\hat{p}), W_{\Delta}\right)= \\
& \quad\left\{M(\hat{p})+\Delta:|\Delta(\omega)| \leq W_{\Delta}(\omega), \forall \omega \in[0,2 \pi]\right\}
\end{aligned}
$$

where, for given $M(\hat{p}) \in \mathcal{P}, W_{\Delta}(\omega)$ has to be chosen such that $\mathcal{M}\left(M(\hat{p}), W_{\Delta}\right)$ is a model set for $P^{o}$, i.e., $\forall \omega \in[0,2 \pi]$ the following condition has to be guaranteed:

$$
W_{\Delta}(\omega) \geq \sup _{P \in F S S}|P(\omega)-M(\omega, \hat{p})|=W_{\Delta}^{*}(\omega, M(\hat{p}))
$$

For given frequency $\omega \in[0,2 \pi]$, exact computation of $W_{\Delta}^{*}(\omega, M(\hat{p}))$ is not easy, but convergent upper and lower bounds are provided by the method presented in Milanese and Taragna (2002). In particular, for given $\nu \in \mathbf{Z}_{1}^{\infty}$ and $m \in \mathbf{Z}_{3}^{\infty}, W_{\Delta}^{*}(\omega, M(\hat{p}))$ can be bounded as:

$$
\underline{W}_{m}^{\nu}(\omega) \leq W_{\Delta}^{*}(\omega, M(\hat{p})) \leq \bar{W}_{m}^{\nu}(\omega)
$$

with the convergence property:

$$
\lim _{\nu, m \rightarrow \infty} \bar{W}_{m}^{\nu}(\omega)=\lim _{\nu, m \rightarrow \infty} \underline{W}_{m}^{\nu}(\omega)=W_{\Delta}^{*}(\omega, M(\hat{p}))
$$

Obviously, $W_{\Delta}^{*}(\omega, M(\hat{p}))$ can be actually evaluated only on a finite set of frequencies. If $P \in K$, the variation rate of $|\Delta(\omega)|$ is bounded by $\max _{0 \leq \omega \leq 2 \pi} \frac{d}{d \omega}|\Delta(\omega)| \leq$ $\frac{L \rho}{(1-\rho)^{2}}$. This information can be used to choose how coarse the gridding has to be for the intersample variation to be negligible.

The error between $P^{o}$ and a given nominal model $M(\hat{p})$ is measured by a weighted $H_{\infty}$ norm:

$$
\left\|P^{o}-M(\hat{p})\right\|_{\infty}^{W_{C}}=
$$

where $W_{C}(\omega)$ is a known positive weighting function, suitably selected according to the intended use of the identified model. For example, if identification is aimed to derive an uncertainty model set for $H_{\infty}$ robust control design, $W_{C}(\omega)$ can be computed so that the identified model optimally approximates the closed loop performance that is underlying the control design, (see, e.g., Milanese et al., 1997). Since $P^{o}$ is only known to belong to $F S S$, the following identification error may be defined.

\section{Definition 3. Model set identification error}

$$
E\left(\mathcal{M}\left(M(\hat{p}), W_{\Delta}^{*}\right)\right)=\sup _{P \in F S S}\|P-M(\hat{p})\|_{\infty}^{W_{C}}
$$

Error $E\left(\mathcal{M}\left(M(\hat{p}), W_{\Delta}^{*}\right)\right)$ gives a measure of the model uncertainty, being the radius of the minimal ball in the $\|\cdot\|_{\infty}^{W_{C}}$ norm with center $M(\hat{p})$ and containing the set of unfalsified systems $F S S$. Exact computation of $E\left(\mathcal{M}\left(M(\hat{p}), W_{\Delta}^{*}\right)\right)$ is hard, but the following convergent upper and lower bounds are provided by the procedure described in Milanese and Taragna (2002):

$$
\begin{aligned}
\underline{E}_{m}^{\nu}\left(\mathcal{M}\left(M(\hat{p}), W_{\Delta}^{*}\right)\right) & \leq E\left(\mathcal{M}\left(M(\hat{p}), W_{\Delta}^{*}\right)\right) \leq \\
& \leq \bar{E}_{m}^{\nu}\left(\mathcal{M}\left(M(\hat{p}), W_{\Delta}^{*}\right)\right)
\end{aligned}
$$

where:

$$
\begin{aligned}
& \underline{E}_{m}^{\nu}\left(\mathcal{M}\left(M(\hat{p}), W_{\Delta}^{*}\right)\right)=\sup _{0 \leq \omega \leq 2 \pi} \underline{W}_{m}^{\nu}(\omega) W_{C}^{-1}(\omega) \\
& \bar{E}_{m}^{\nu}\left(\mathcal{M}\left(M(\hat{p}), W_{\Delta}^{*}\right)\right)=\sup _{0 \leq \omega \leq 2 \pi} \bar{W}_{m}^{\nu}(\omega) W_{C}^{-1}(\omega)
\end{aligned}
$$

The model set uncertainty can be minimized by appropriately choosing the nominal model $M(\hat{p})$. The best model set corresponds to $M\left(p^{*}\right)$, where

$$
p^{*}=\arg \min _{p} E\left(\mathcal{M}\left(M(\hat{p}), W_{\Delta}^{*}\right)\right)
$$

can be found by means of iterative nonlinear optimization algorithms. The model $M\left(p^{*}\right)$, where $p^{*}$ is solution of (6), is the so called "conditional center" of $F S S$. Finding conditional centers is hard, (see, e.g., Kacewicz, 1999) for some results in the case that the class of models $M(p)$ is linear in the parameters, e.g., represented by FIR, Laguerre, Kautz or orthonormal basis functions. These models are essentially with fixed poles and the problem arises of choosing "good" basis, i.e., "good" model poles, since this choice affects, for given model order, the identification accuracy. On the other hand, models nonlinear in the parameters, able to arrange also their poles, may improve the identification accuracy, but make problem (6) nonconvex, and trapping in local minima may take place. 
In such a case, the choice of a good starting point to be used by iterative nonlinear optimization algorithms for solving (6) is very important. Indeed, very good results may be achieved by using the procedure proposed in Milanese and Taragna (2000):

- compute the "nearly optimal" $F I R_{\nu}$ model $M_{\nu}^{\text {no }} \in F S S$, whose impulse response is obtained as solution of a suitable linear programming problem;

- compute reduced order models $M_{n}^{r}(\hat{p})$ of $M_{\nu}^{n o}$ by means of Hankel norm approximation or balanced realization and SVD truncation.

This is due to the well-known relationship between Hankel and $H_{\infty}$ norms, and to the fact that $M_{n}^{r}$ is a "nearly central" projection model in the Hankel norm. Indeed, central projection models are known to provide good approximations of conditionally central models (Kacewicz, 1999).

The described Set Membership $H_{\infty}$ identification procedure can be performed using the MATLAB tool SMID presented in Malan et al. (2002).

The above method allows to derive "hard" uncertainty models, making use of the knowledge of deterministic constraints on measurement noise. If probabilistic information on the noise is available, "soft" uncertainty models, guaranteeing the inclusion property (1) with assigned probability, can be estimated using the method of Milanese and Taragna (1999).

\section{ROBUST IMC DESIGN AND GUARANTEED CLOSED LOOP PERFORMANCES}

The robust Internal Model Control (IMC) design is carried out in the continuous-time setting, using the continuous-time uncertainty model derived by applying the bilinear transformation to the discrete-time uncertainty model identified with the method presented in the previous section.

It is well known that, in the case of stable plant, the $I M C$ structure (see figure 1 with $\Delta=0$ ) parameterizes, in terms of a stable transfer function $Q$, all the controllers $C$ which provide stability of the closed loop system with unity negative feedback:

$$
C=\frac{Q}{1-Q M}
$$

The design of the controller $C$ is, then, equivalent to the design of its parameterization $Q$, but some remarkable advantages, such as stability preservation, are obtained by using the IMC implementation in presence of saturating actuators (see Morari and Zafiriou, 1989; Canale and Milanese, 2001).

Now consider the IMC structure of figure 1, with model uncertainty $\Delta \neq 0$, and define as usual the sensitivity and the complementary sensitivity functions:

$$
S(s)=\frac{Y(s)}{D(s)} \quad T(s)=\frac{Y(s)}{R(s)}
$$

IMC methods have been investigated in past years for designing robust control in the face of unmodeled dynamics (Morari and Zafiriou, 1989; Lee et al., 1993; Campi et al., 1994). It is straightforward to compute

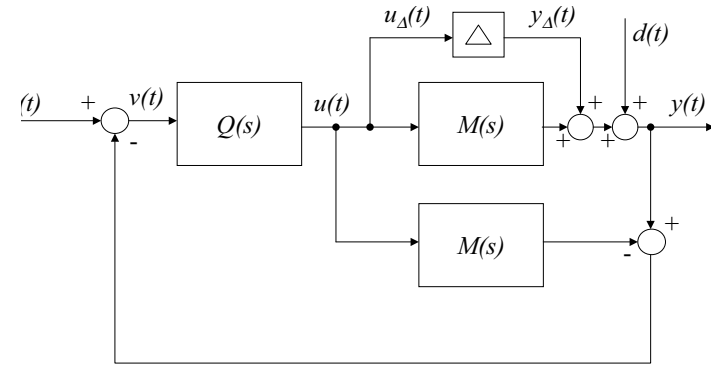

Fig. 1. IMC structure

that, if the closed loop is robustly stable in the face of model uncertainty $\Delta(s)$, and

$$
1-Q M=0
$$

then $S(s)=0$ and $T(s)=1$ ("perfect control") for all stable $\Delta(s)$. However, it is also usually required that $Q$ is proper and stable and these requirements cannot be met by the choice $Q=M^{-1}$ if $M$ is strictly proper or nonminimum-phase.

In standard IMC approach, the parameter $Q$ is obtained as $Q=\widetilde{Q} F$ and a two stage design procedure is adopted. First, a nominal design (i.e. $\Delta(s)=0$ ) is performed computing $\widetilde{Q}$ by minimizing $\|(1-M \widetilde{Q}) v\|_{2}$ for some canonical signal $v=r-d$ (typically step signal). Then, the filter $F$ is chosen as a rational function such that $Q$ is proper and nominal closed loop is internally stable. The filter $F$ is designed depending on a parameter $\lambda$, whose value is related to the nominal closed loop bandwidth. Then, on the basis of model uncertainty, the parameter $\lambda$ is chosen to give the maximal bandwidth compatible with stability and performance of the actual $(\Delta(s) \neq 0)$ closed loop, looking at the quality of the actual response to the considered canonical reference signals.

In this paper, the $I M C$ design problem is reformulated in terms of $H_{\infty} / \mu$-optimization. Considering the "perfect control" ideal target (7), a cost function is defined in terms of the $H_{\infty}$ norm of $\left(1-Q M_{n}(\hat{p})\right)$. Moreover the robust stability constraint imposed by the Small Gain Theorem requires $\|Q \Delta\|_{\infty}<1$, being $-Q$ the transfer function from $y_{\Delta}$ to $u_{\Delta}$ (see figure 1 ), for which a sufficient condition is $\left\|Q W_{\Delta}^{*}(\omega)\right\|_{\infty}<1$. Then $Q$ is obtained as solution of the following constrained optimization problem:

$$
\begin{gathered}
Q^{*}=\arg \min _{Q \in \mathcal{R} \mathcal{H}} \sup _{\infty \mid \leq W_{\Delta}^{*}}\left\|\left[1-Q\left(M_{n}(\hat{p})+\Delta\right)\right] W\right\|_{\infty} \\
\text { s.t. }\left\|Q W_{\Delta}^{*}(\omega)\right\|_{\infty}<1
\end{gathered}
$$

where $W$ is a rational weighting function defined on the basis of the spectral features of signals $r(t)$ and $d(t)$.

The solution of this problem can be obtained formulating the problem as the following $\mu$-synthesis problem:

$$
Q_{\mu}^{*}=\arg \min _{Q \in \mathcal{R} \mathcal{H}_{\infty}} \sup _{\omega \in \mathbf{R}} \mu(\omega)
$$

where $\mu=\left|W_{r} Q\right|+\left|\left(1-Q M_{n}\right) W\right|$ and $W_{r}(s)$ is a stable rational function tightly overbounding $W_{\Delta}^{*}$ (see Scheid and Bayard, 1995, for a systematic approach for such overbounding).

Once completed the design procedure, the uncertainty model can be used to calculate the guaranteed closedloop performances, that is to calculate the frequency 
bounds for the considered performance, for given $\omega \in$ $\mathbf{R}^{+}, Q^{*} \in \mathcal{H}_{\infty}$ and $\mathcal{M}\left(M(\hat{p}), W_{\Delta}\right) \subseteq \mathcal{P}$ :

$$
\begin{gathered}
\underline{H}(\omega, Q, \mathcal{M}) \leq|\tilde{H}(j \omega, Q, P)| \leq \bar{H}(\omega, Q, \mathcal{M}), \\
\forall P \in \mathcal{M}\left(M(\hat{p}), W_{\Delta}^{*}\right)
\end{gathered}
$$

where

$$
\begin{aligned}
& \bar{H}(\omega, Q, \mathcal{M})=\sup _{P \in \mathcal{M}\left(M(\hat{p}), W_{\Delta}^{*}\right)}|\tilde{H}(j \omega, Q, P)| \\
& \underline{H}(\omega, Q, \mathcal{M})=\inf _{P \in \mathcal{M}\left(M(\hat{p}), W_{\Delta}^{*}\right)}|\tilde{H}(j \omega, Q, P)|
\end{aligned}
$$

and $\tilde{H}(\cdot)$ are closed loop transfer functions of interest. Note that for some functions (e.g., sensitivity) such bounds can be computed exactly, while for other functions (e.g., complementary sensitivity) only approximated (though tight) values can be obtained, (see, e.g., Malan et al., 2002).

It can be noted that, although the controller is directly designed in order to provide robust performances, this a posteriori robustness analysis is necessary to verify if the obtained closed loop guarantees the fulfillment of the performance requirements when the original weight $W$ and $W_{\Delta}^{*}$ are considered instead of their rational approximation used in the design. Moreover, it is straightforward that such a computation can be applied to any given stabilizing controller. Thus, the computation of the guaranteed performance obtained with a reduced order controller, provided by standard order reduction technique, allow us to suitably trade off between controller complexity and robust performance fulfillment.

The computation of the guaranteed performance bound can be performed by using the MATLAB tool GPC presented in Malan et al. (2002).

\section{EXAMPLE}

Robust control from data of the following nonminimumphase continuous-time system is considered:

$P(s)=\frac{-0.0609 s^{4}-0.4871 s^{3}-0.4871 s^{2}+1.9482 s+2.9224}{s^{4}+0.4311 s^{3}+2.6764 s^{2}+0.4384 s+0.1412}$

The available experimental information is made up by $N=1100$ samples of the system output to a PRBS input of unitary amplitude, using $T_{s}=1 \mathrm{~s}$ as sampling time. These samples are corrupted by a pointwise bounded noise $e^{N}$ with $\left|e_{i}\right| \leq 4, i=0,1, \ldots, 1099$. This data set is used to identify a discrete-time uncertainty model guaranteeing to contain the discrete-time system $P^{o}$, consisting of the plant and the input and output sampling devices.

The prior information assumed on $P^{o}$ is $L=6$ and $\rho=0.93$, which has been validated using Theorem 2 in Milanese and Taragna (2000). This is a quite "safe" prior assumption, since stronger assumptions are still not invalidated by data, e.g., $L=6$ and $\rho=0.91$, or $L=5$ and $\rho=0.93$.

Different nominal models have been identified, with their corresponding model sets:
- a 150-th order FIR model $M_{150}^{n o}$ provided by the "nearly optimal" algorithm;

- approximations of $M_{150}^{n o}$ of order $2 \div 6$, denoted by $M_{2} \div M_{6}$, provided by the procedure described in section 3 .

The computations of the $\sup _{0<\omega<2 \pi}$ required in (5), with $W_{C}(\omega)=1 \forall \omega \in[0,2 \pi]$, have been carried on by gridding $\omega$ on a sufficiently coarse set of frequencies. Indeed, the value sets $V(\omega)$ have been computed on a set $T_{\omega}$ of 500 equispaced frequencies in the interval $[0, \pi]$.

The obtained values of the lower and upper bounds on the identification error $E(\mathcal{M})$ for the identified model sets $\mathcal{M}_{150}^{n o}$ and $\mathcal{M}_{n}$ (with nominal model $M_{n}$ ), for $n=2, \cdots, 6$. are reported in the following table:

\begin{tabular}{|c|c|c|}
\hline $\mathcal{M}$ & $\underline{E}_{16}^{150}(\mathcal{M})$ & $\bar{E}_{16}^{150}(\mathcal{M})$ \\
\hline \hline $\mathcal{M}_{150}^{n o}$ & 3.57 & 3.61 \\
\hline \hline $\mathcal{M}_{2}$ & 6.49 & 6.52 \\
\hline $\mathcal{M}_{3}$ & 5.31 & 5.34 \\
\hline $\mathcal{M}_{4}$ & 3.48 & 3.49 \\
\hline $\mathcal{M}_{5}$ & 3.48 & 3.49 \\
\hline $\mathcal{M}_{6}$ & 3.48 & 3.49 \\
\hline
\end{tabular}

From these results it is seen that model sets of order higher than 4 do not allow to improve the identification error. Then the control design is performed on model sets $\mathcal{M}_{4}$. The magnitude frequency response of the identified model set $\mathcal{M}_{4}$ is reported in figure 2 and compared with the actual system $P^{o}$.

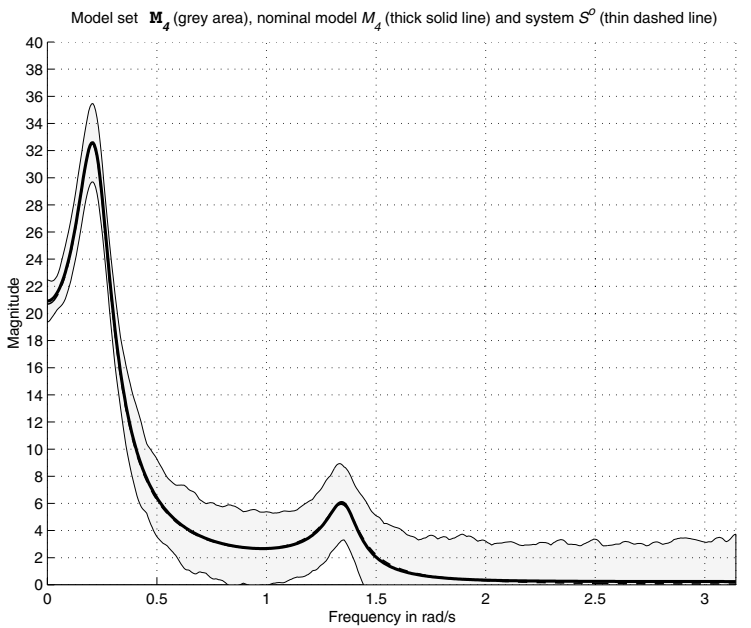

Fig. 2. Frequency response of model sets $\mathcal{M}_{4}$

The nominal reduced order model $M_{4}$, mapped in $s$-domain using inverse bilinear transformation with a sampling time $T_{s}=1 s$, and the corresponding information about identification error are used for computing $Q_{\mu}^{*}$ as described in section 4 .

Attenuation of $50 d B$ of disturbance $d(t)$ in the frequency band $\omega \in\left[0,10^{-4}\right] \mathrm{rad} / \mathrm{s}$ and maximum sensitivity peak of $6 \mathrm{~dB}$ are the considered performance specification. Then the following performance weighting function is assumed:

$$
W=\left\{\begin{aligned}
312.5 & \text { for } \omega \in\left[0,10^{-4}\right] \mathrm{rad} / \mathrm{s} \\
0.5 & \text { for } \omega \in\left[10^{-4}, \infty\right] \mathrm{rad} / \mathrm{s}
\end{aligned}\right.
$$


In order to use the $H_{\infty} / \mu$-optimization algorithm available under MATLAB the two rational functions $W_{r}(s)$ and $W_{s}(s)$ mentioned in section 4 must be used respectively in place of $W_{\Delta}^{*}$ and $W$. Considering that $W_{\Delta}^{*}$ is approximately constant, a suitable choice for $W_{r}$ is the following:

$$
W_{r}(s)=\bar{E}_{16}^{150}\left(\mathcal{M}_{4}\right)
$$

A reasonable (low-order) rational approximation of $W$ is:

$$
W_{s}(s)=\frac{0.50119(s+0.3786)}{s+0.0006}
$$

The optimization (8) was actually performed using D$\mathrm{K}$ iteration algorithm. Starting with the initial scaling weighting matrix $D$ set to identity, the procedure provides both robust stability and robust performance requirements fulfillment (i.e., $\sup _{\omega \in \mathbf{R}} \mu(\omega)<1$ ) after 2 D-K iterations. The obtained 12th order controller $C_{\mu}^{*}=Q_{\mu}^{*} /\left(1-M_{4} Q_{\mu}^{*}\right)$ is then reduced to a 5 th order controller $C_{r}^{*}=Q_{r}^{*} /\left(1-M_{4} Q_{r}^{*}\right)$ using standard model reduction techniques.

Figure 3 shows the guaranteed sensitivity upper and lower bounds for model sets respectively obtained with the full order controller $Q_{\mu}^{*}$ and the reduced order controller $Q_{r}^{*}$. The analysis of these results provides interesting information to the designer about the tradeoff between closed loop performances and controller complexity. As a matter of fact, in the example considered here the computation of the guaranteed sensitivity performances show that a 5 th order controller can be used instead of the full order controller provided by the D-K algorithm without any performance degradation. It is also interesting to note that in Malan et al. (2001) an optimal $H_{\infty}$ controller was designed, for the same problem considered in this example, looking for nominal performance and robust stability optimization. Such controller, though of the same order of the reduced order one $C_{r}$ proposed in this work, did not provide robust performances.

\section{REFERENCES}

Campi, M., W. S. Lee and B. D. O. Anderson (1994). New filters for internal model control design. International Journal of Robust and Nonlinear Control 4, 757-775.

Canale, M. and M. Milanese (2001). Robust control of linear systems in presence of input saturation and unmodelled dynamics. In: Proc. of the 40th IEEE Conference on Decision and Control. Orlando, FL. pp. 4248-4253.

Giarré, L., M. Milanese and M. Taragna (1997). $H_{\infty}$ identification and model quality evaluation. IEEE Transactions on Automatic Control AC-42(2), 188-199.

Hakvoort, R. G. and P. M. J. Van den Hof (1995). Consistent parameter bounding identification for linearly parametrized model sets. Automatica 31(7), 957-969.

Kacewicz, B. (1999). Worst-case conditional system identification in a general class of norms. Automatica 35(6), 1049-1058.

Lee, W. S., B. D. O. Anderson and R. L. Kosut (1993). A new approach to adaptive robust control. International Journal on Adaptive Control and Signal Processing 7, 183-211.

Mäkilä, P. M., J. R. Partington and T. K. Gustafsson (1995). Worst-case control-relevant identification. Automatica 31(12), 1799-1819.

Malan, S., M. Milanese and M. Taragna (2002). MATLAB tools for SM $H_{\infty}$ identification and guaranteed control performance computation. In: Proc. of the 41st IEEE Conference on Decision and Control. Las Vegas, NV. pp. 4203-4208.

Malan, S., M. Milanese, D. Regruto and M. Taragna (2001). Robust control from data via uncertainty model sets identification. In: Proc. of the 40th IEEE Conference on Decision and Control. Orlando, FL. pp. 2686-2691.
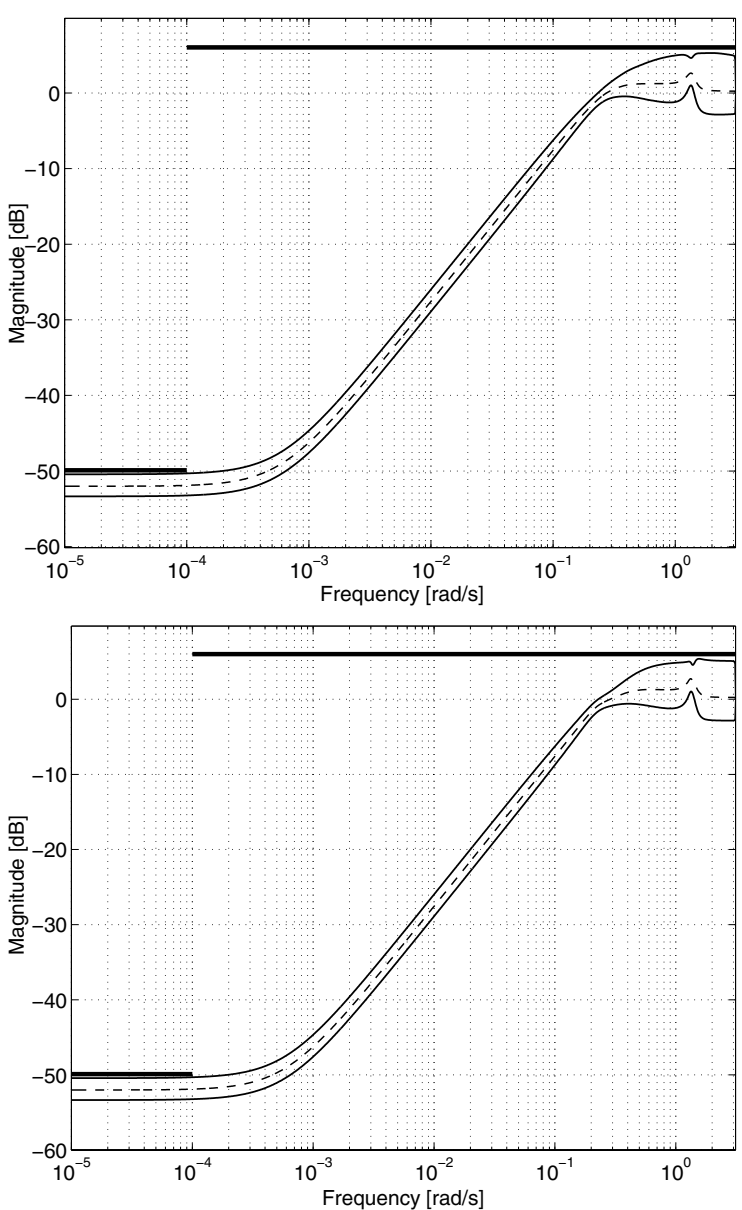

Fig. 3. Guaranteed bounds on sensitivity (thin), nominal sensitivity (dashed) and performance weight $W$ with controller $Q_{\mu}^{*}$ (above) and $Q_{r}^{*}$ (below)

Milanese, M. and A. Vicino (1993). Information-based complexity and nonparametric worst-case system identification. Journal of Complexity 9, 427-446.

Milanese, M. and M. Taragna (1999). $H_{\infty}$ identification of "soft" uncertainty models. Systems \& Control Letters 37(4), 217228 .

Milanese, M. and M. Taragna (2000). Set Membership identification for $H_{\infty}$ robust control design. In: Proc. of 12th IFAC Symposium on System Identification SYSID 2000. Santa Barbara, CA.

Milanese, M. and M. Taragna (2002). Optimality, approximation, and complexity in Set Membership $H_{\infty}$ identification. IEEE Transactions on Automatic Control AC-47(10), 1682-1690.

Milanese, M., M. Taragna and P. M. J. Van den Hof (1997). Closedloop identification of uncertainty models for robust control design: a set membership approach. In: Proc. of the 36th IEEE Conference on Decision and Control. San Diego, CA. pp. 2447-2452.

Morari, M. and E. Zafiriou (1989). Robust process control. Prentice Hall. Englewood Cliffs, NJ.

Ninness, B. and G. C. Goodwin (1995). Estimation of model quality. Automatica 31(12), 1771-1797.

Scheid, R. E. and D. S. Bayard (1995). A globally optimal minimax solution for spectral overbounding and factorization. IEEE Transactions on Automatic Control AC-40(4), 712-716.

Special Issue on System Identification for Robust Control Design, (1992). IEEE Transactions on Automatic Control AC37(7), 899-974.

Venkatesh, S. R. and M. A. Dahleh (2001). On system identification of complex systems from finite data. IEEE Transactions on Automatic Control AC-46(2), 235-257.

Zhou, T. and H. Kimura (1995). Structure of model uncertainty for a weakly corrupted plant. IEEE Transactions on Automatic Control AC-40(4), 639-655. 